\title{
Table ronde. Le Nouveau Roman : passé, présent,
} futur

Tom Bishop, François Jost, Robert Pinget, Alain Robbe-Grillet, Michel Rybalka, Nathalie Sarraute, Claude Simon et Monique Wittig

\section{(2) OpenEdition \\ 1 Journals}

Édition électronique

URL : http://journals.openedition.org/ccs/2632

ISSN : 2558-782X

\section{Éditeur :}

Presses universitaires de Rennes, Association des lecteurs de Claude Simon

\section{Édition imprimée}

Date de publication : 30 septembre 2019

Pagination : 37-54

ISBN : 978-2-7535-7795-4

ISSN : $1774-9425$

Référence électronique

Tom Bishop, François Jost, Robert Pinget, Alain Robbe-Grillet, Michel Rybalka, Nathalie Sarraute, Claude Simon et Monique Wittig, «Table ronde. Le Nouveau Roman : passé, présent, futur », Cahiers Claude Simon [En ligne], 14 | 2019, mis en ligne le 30 septembre 2020, consulté le 10 octobre 2020. URL : http://journals.openedition.org/ccs/2632 


\title{
TABLE RONDE LE NOUVEAU ROMAN : PASSÉ, PRÉSENT, FUTUR
}

\author{
Tom BISHOP, François JOST, Robert PINGET, Alain ROBBE-GRILLET, \\ Michel RYBALKA, Nathalie SARRAUTE, Claude SIMON et Monique WITTIG
}

Les absents ont toujours tort et Jean Ricardou a dî en faire la douloureuse expérience à la suite de ce colloque-bilan intitulé "Three Decades of the French New Novel " qu'organise Tom Bishop avec Lois Oppenheim ${ }^{1}$ à l'université de New York du 30 septembre au 2 octobre 1982. Quoique physiquement absent, Ricardou se trouve pourtant au cour de maints échanges pendant l'une des deux tables rondes de clôture qui réunit les romanciers Robert Pinget, Alain Robbe-Grillet, Nathalie Sarraute, Monique Wittig et Claude Simon, ainsi que les universitaires François Jost et Tom Bishop ${ }^{2}$. Et c'est peu dire qu'il fait l'objet de réflexions peu amènes. On peut même considérer que ce colloque scelle en un sens la fin de ce qui fut appelé Nouveau Roman.

Il faut dire qu'au début des années 1980, d'une part les romanciers ont pris leurs distances avec Ricardou, et d'autre part, les querelles suscitées par les innovations formelles des "nouveaux romanciers " sont apaisées et paraissent un peu lointaines, chacun ayant poursuivi et mûri son æuvre singulière. Tel Claude Simon, dont on salue unanimement Les Géorgiques comme un "très grand livre ", en septembre 1981. La même année, Robbe-Grillet fait paraître Djinn et, un an plus tôt, Nathalie Sarraute publie L'Usage de la parole. Trois romans nouveaux, certes, mais trois écritures fort différentes. Le retentissement des Géorgiques incite d'ailleurs la presse à sinterroger sur le devenir du "nouveau roman ", dix ans après le premier

1. Les actes du colloque ont été publiés par L. Oppenheim (dir.), Three Decades of the French New Novel, Urbana et Chicago, University of Illinois Press, 1986.

2. Également présent, l'universitaire Michel Rybalka n’intervient pas dans les débats. 
colloque qui lui a été consacré à Cerisy-la-Salle, à l'été 1971. Aux États-Unis, où l'accueil de la littérature française innovante a été très enthousiaste, c'est aussi l'heure du bilan avec ce colloque qui réunit des critiques, des universitaires et des écrivains français et américains ${ }^{3}$. Un bilan et une mise en perspective, au cours desquels certes Alain Robbe-Grillet rappelle les convergences littéraires des néo-romanciers et le rôle déclencheur joué par Le Vent, en 1957, mais où chacun des écrivains, dans la suite du colloque, vient souligner la spécificité de sa propre poétique.

Ainsi, les "Réflexions sur le roman" de Claude Simon, que le romancier présente comme celles d'un "autodidacte ", se gardent de toute généralisation et de toute prétention à un savoir théorique. C'est en praticien que l'écrivain parle de son écriture, qu'il s'efforce de dissiper quelques "malentendus" sur la notion de fragmentation, qu'il souligne l'importance majeure de la composition, régie par la recherche de "l'établissement de rapports avant tout qualitatifs ", qu'il évoque le rôle essentiel pour lui de la leçon de Cézanne cherchant à rendre compte de "sensations ", et qu'il insiste, à propos des Géorgiques, sur la continuité du principe d' "incertitude " toujours à l'œuvre dans ses livres, aussi bien que dans ceux de ses compagnons 4 .

Le colloque s'achève par deux tables rondes, dont l'une invite les romanciers français à confronter leurs points de vue et à porter un regard perspectif sur ce mouvement né un quart de siècle plus tôt. C'est cette table ronde que nous publions pour la première fois en français ${ }^{5}$, une table ronde qui a fait date. Le recul et la distance permettent d'envisager l'impact de la "césure " que représente le Nouveau Roman sur l'évolution du genre et sur les écrivains de la génération suivante, européens ou américains. Car si le "nouveau roman " n’a mis fin ni

3. John Barth, Jonathan Baumbach, Robert Coover et John Hawkes ont pris part à la table ronde finale (voir "American Parallels : An Afterword ", dans Three Decades of the French New Novel, éd. cit., p. 195-209).

4. C. Simon, conférence sans titre, dans Three Decades of the French New Novel, éd. cit., p. 71-86. À peu près à la même époque, Simon prononce deux des conférences, "Le Poisson cathédrale " (1980) et "L'absente de tous bouquets " (1982) qui abordent des problématiques similaires et qui seront publiées dans Quatre conférences (textes établis et annotés par Patrick Longuet, Minuit, 2012).

5. Le texte a initialement été publié dans Three Decades of the French New Novel sous le titre "The New Novel - Past, Present, Future : A Rondtable" (éd. cit., p. 179-194). Nous remercions tout particulièrement Lois Oppenheim et Tom Bishop d'en avoir permis la réédition, ainsi que François Jost, Mireille Calle-Gruber, Olivier Corpet et Luciola Pinget, ces trois derniers en qualité d'ayant-droit respectivement des œuvres d'Alain Robbe-Grillet, de Claude Simon et de Robert Pinget. Nous sommes également redevables à Alastair B. Duncan, qui a trouvé à l'IMEC dans les archives Alain Robbe-Grillet la version française de cette table ronde.

6. Voir l'ouvrage de synthèse récemment réédité (et augmenté) de F. Dugast-Portes, Le Nouveau Roman, Une césure dans l'histoire du récit, préface de F. Dosse, Rennes, PUR, 2018, notamment, sur le colloque de New York, p. 103-107. 
à l'intrigue ni aux personnages, il a évolué et ouvert très largement l'éventail des formes de narration et de description en ébranlant les frontières génériques. Ce souci d'ouverture et de liberté se fait particulièrement entendre quand survient dans les échanges la question du discours théorique, de ses limites et de ses dangers, la plupart des romanciers s'accordant à en reconnaître les apports, tout en rejetant unanimement (à l'exception de Robert Pinget) et avec vigueur les excès d'un Ricardou qui est passé "de la proscription à la prescription » et qui entend réguler ce qui ne peut l'être, la création littéraire. Soudain, l'hégémonisme ricardolien qui régnait sur les colloques de Cerisy dans les années 1970 paraît bien loin 7 .

Tom Bishop : Personnellement, j'ai été très frappé par certaines choses, ces trois derniers jours, notamment le fait que nous ayons trouvé, que vous vous soyez trouvé surtout, des points communs plutôt que des éléments qui vous séparent. Robert Pinget a parlé de solidarité parmi les écrivains du Nouveau Roman. Je pense que c'est ce qui ressort actuellement. En tout cas, c'est ce qui me semble ressortir ici. C'est peut-être une tolérance, mais je pense que c'est plutôt une estime que vous, les Nouveaux Romanciers, semblez avoir les uns pour les autres, et que vous avez sans doute toujours eue.

Cela est peut-être dû au fait que pendant très longtemps on a surtout voulu souligner ce qui vous sépare. C'est-à-dire que pendant un certain temps, très longtemps, et tout cela a éclaté autour de Cerisy, il y avait une tendance à vous définir et à vous laisser définir par les théoriciens. Il me semble que cette époque est révolue et qu'aujourd'hui vous parlez tous de votre propre œuvre. C'est-à-dire que vous tendez tous à parler de votre œuvre sans penser avant toute chose au point de vue critique et à tout le cadre théorique, toute la superstructure théorique qui a pesé d'un certain poids pendant une période assez longue. Je vois là une libération dans la manière que vous avez tous de considérer votre ouvre. C'est peut-être un certain détachement olympien qui vient du fait que le Nouveau Roman est plus établi, mais j'aimerais vous demander si en effet vous avez l'impression de vous être séparés du reflet que vous renvoyait la critique des années soixante et soixante-dix?

7. Voir également dans ce numéro l'éclairante " Note supplémentaire " d'Anthony C. Pugh sur le sujet, p. 131-134. 
Claude Simon : En ce qui me concerne, le reflet que la critique renvoyait de nous, si vous voulez parler de la critique au jour le jour qui est, comme on sait, une critique d'humeur, je ne me suis jamais très (ou trop?) préoccupé de ses attaques, la plupart du temps dérisoires. Quant à la critique au second degré, la plus sérieuse, la critique que l'on pourrait appeler " théoricienne " (et je pense que c'est à Ricardou que vous faites allusion), il me semble que c'est plutôt lui qui s'est séparé de nous - ou tout du moins de moi. Tout en ne cessant de considérer ses travaux théoriques comme des plus estimables, il m’apparaît qu'il a poussé à l'extrême un esprit de radicalisation assez stérilisant pour la pratique et qui s'est retourné contre lui-même...

Alain Robbe-Grillet : Je crois qu'on pourrait dire que le rôle de Ricardou a été très positif car le rôle de la théorie pour un écrivain n'est pas du tout de le réconforter. Ce n'est pas qu'il aurait fait des ouvres d'abord et puis qu'il aurait été renforcé par des fortifications théoriques, par la défense de l'œuvre en question. C'est peut-être comme cela que Ricardou le concevait, mais pour nous, écrivains, la théorie a toujours joué un rôle complètement différent. Il s'agissait de nous chasser hors de nous-mêmes, plutôt que de nous conforter dans des positions acquises. C'est-à-dire que tout ce qui peut être théorisé est pratiquement sans intérêt pour le créateur. De même que l'œuvre d'art commence là où le sens s'arrête, de même on peut dire que ce que je cherche à faire commence là où la théorie de ce que je cherche à faire s'arrête. Par conséquent, la théorie est très intéressante parce que cela va justement montrer jusqu'où le sens peut s'avancer et où va commencer ce qui n'est plus le sens formel du formaliste mais qui est justement l'œuvre à faire. Ce n'est pas l'œuvre qu'il a faite. Et la théorisation ne peut se faire que sur ce qui existe déjà.

Quant à moi, je sais que je ne renie pas du tout les travaux théoriques de Ricardou, même si je les trouve déments. Cette démence fait justement partie de quelque chose qui sans cesse fascine l'écrivain. "Et aujourd'hui, dit Tom Bishop, on s'est éloigné de... " Non! Il y a des théories qui ont fait leur temps. Et pas seulement les théories formalistes ricardoliennes. C'est toute une époque de théorisation. C'est une époque où la théorie a beaucoup joué, et souvent, cela agaçait les Américains que la théorie joue un tel rôle en France. Je suis, il me semble, très français de ce point de vue : j'aime les théories. Bien entendu, je ne tiens pas du tout à les respecter, mais je suis de formation scientifique et les théories scientifiques m'ont toujours passionné. Jamais un scientifique ne pense qu'une théorie doive être vraie ou fausse. Cela 
n'existe pas en science. Une théorie n'est pas vraie ou fausse; une théorie est productive ou non. Il y a des théories qui, à un moment donné, ont produit quelque chose, ne serait-ce que justement pour nous chasser hors de nousmêmes, nous chasser hors des textes que nous avions écrits et qu'on pouvait déjà théoriser.

Tom Bishop : Mais le fait qu'aujourd'hui ces théories n'ont plus la même...

Alain Robbe-Grillet : Mais nous en préparons d'autres! Méfiez-vous!

Nathalie SARRAUte : En ce qui concerne Ricardou, j'ai toujours considéré que ses théories, du moins la plupart d'entre elles, étaient insoutenables. Je crois que cela n'avait aucune importance pour les écrivains parce que, quand ils écrivent, ils ne pensent plus aux théories. Je crois cependant que les points de vue de Ricardou étaient dangereux pour les lecteurs, parce que nous sommes tous des lecteurs, et moi, en tant que lectrice, si j'avais dû lire des livres en suivant les principes de Ricardou, j'aurais cessé de lire. Il me serait impossible de lire un livre de Claude Simon en y cherchant le mot « jaune » à la page soixante-sept et à la page cent vingt-six, même si c'était la règle du jeu et si Claude Simon avait écrit son livre pour qu'on y trouve ce mot, ce que je ne crois pas d'ailleurs. C'était cela le danger des théories de Ricardou : elles risquaient de détourner de la lecture.

Monique Wittig : Je voudrais rappeler qu'Alain Robbe-Grillet, dans Pour un Nouveau Roman, commence son livre en disant qu'il n'est pas un théoricien. Cependant, il explique les problèmes qu'il a eus au fur et à mesure de ses écrits. C'est la même chose pour Nathalie Sarraute. Dans les deux cas, il était nécessaire de se défendre devant une certaine presse qui interprétait de travers ce qui se passait. Je sentais exactement la même chose tout à l'heure quand je réagissais à l'écriture féminine ${ }^{8}$. Maintenant, dès qu'une femme parle, on lui colle l'écriture féminine et pour moi, le concept d'écriture féminine est un paradoxe qu'il est absolument impossible de soutenir.

Alain Robbe-Grillet : Ce que Monique Wittig fait remarquer est très juste. C'est vrai que Nathalie Sarraute, comme moi, a publié des écrits à

8. Monique Wittig fait référence à son échange avec Leon S. Roudiez lors de la discussion qui suit la conférence de ce dernier (Three Decades of the French New Novel, éd. cit., p. 175). 
caractère théorique. Mais ces écrits à caractère théorique ne se sont jamais considérés comme une théorie du roman et a fortiori comme la théorie du roman. C'est-à-dire qu'un théoricien, c'est quelqu'un qui bâtit une totalité. C'est quelqu'un qui fait entrer dans une forme close toutes ses préoccupations et, par conséquent, qui édicte des vérités normatives concernant le domaine de la littérature par exemple. Et il est évident que Nathalie Sarraute, dans L'Ėre du soupçon, ou moi-même, dans Pour un Nouveau Roman, nous n'avons jamais fait cela.

En terminant la première séance ici, François Jost est allé dans cette direction-là quand il a fait remarquer qu'on était passé avec Ricardou de la proscription à la prescription. C'est-à-dire que ce que nous avons théorisé d'abord, Nathalie Sarraute et moi, c'était le langage de la critique au pouvoir. Nous avons analysé des expressions, des mots, des critères que nous trouvions dans la presse au pouvoir et tout ce que Nathalie Sarraute a publié dans L'Ėre $d u$ soupçon était en quelque sorte des analyses de ce qu'on nous reprochait de ne pas faire. Évidemment, c'est de la proscription. C'est-à-dire que nous nous opposions à ces critères.

En revanche, il est vrai que la période de Ricardou a été une période où tout d'un coup quelqu'un a voulu prescrire. C'est-à-dire dire "C'est cela qu'il faut faire. " Évidemment c'était tout à fait contraire à notre idée à nous du Nouveau Roman puisque quand nous nous étions groupés, Nathalie Sarraute et moi au début et puis tous nos amis, c'était justement sous le sigle de notre liberté. C'est-à-dire que ce que nous réclamions pour chaque écrivain, c'était la liberté d'inventer le roman, chacun pour soi et à chaque roman qu'il écrivait.

Tout écrivain qui invente le roman pour moi est un « Nouveau Romancier ». Et je crois que c'est Nathalie Sarraute aussi qui, déjà au début, faisait remarquer que le mot " formaliste " était appliqué à tort aux gens qui inventaient des formes nouvelles. Car au contraire, les formalistes, ce sont ceux qui reproduisent des formes qui existent déjà. Et sur ce point donc, il n'y avait pas du tout de théorisation prescriptive mais seulement une analyse descriptive de ce que disait la critique au pouvoir.

Tom Bishop : Alain Robbe-Grillet, vous avez dit hier ou avant-hier que la révolution que devait être le Nouveau Roman n'a pas abouti et que la littérature finalement n'a pas été bouleversée par vous au pluriel. 
Alain RobBe-Grillet : Non, elle a été bouleversée, mais elle n'a pas abouti parce que la liberté ne peut pas être une institution. C'est-à-dire que si le Nouveau Roman avait abouti, Ricardou aurait en somme pris le pouvoir et nos romans auraient été institutionnalisés. C'est ce que Staline a fait, si vous voulez, pour le marxisme : c'est-à-dire transformer en dogmatisme ce qui au départ était un mouvement vers, un mouvement de, quelque chose qu'on ignore, une espèce de projet qui ne se connaît pas lui-même et qui s'aventure dans une direction qui l'excite. Il n'a donc pas abouti, heureusement. S'il avait abouti, nous aurions cessé d'écrire et tout le monde aurait cessé d'écrire. Heureusement, dis-je, car ce mouvement de se chasser sans cesse hors de soi ne peut pas avoir de fin. Le plus grand danger pour un écrivain, c'est de s'asseoir sur ses conquêtes, c'est-à-dire qu'il a écrit tel livre qui a plu, et qu'il va maintenant en faire un autre pareil.

Vous savez qu'on m'a toujours reproché à moi personnellement d'écrire moins bien que je ne le faisais l'année précédente. Les critiques qui avaient méprisé du haut de leur grandeur La Jalousie, au moment où je publiais Projet pour une révolution à New York, se sont mis, par exemple, à dire qu'il était dommage que je n'aie pas continué dans cette belle voie qu'était La Jalousie. Or justement, ce qui est important pour un artiste, c'est de décevoir son public, c'est-à-dire de l'empêcher de se tranquilliser dans son fauteuil. Et le fait que le Nouveau Roman ne s'est pas institutionnalisé, je l'ai présenté comme le fait qu'il n'a pas abouti, mais cette défaite, c’est sa vie même.

Tom Bishop : Est-ce que cette façon d'aboutir ou de ne pas aboutir, de bouleverser ou pas, n'implique pas aussi une autre génération, une génération qui aurait été celle d'après vous et qui ferait des romans liés d'une certaine sorte à ce que vous avez fait? Est-ce que vous avez l'impression qu'il y a des auteurs qui ont suivi d'une manière ou d'une autre ce que vous avez essayé de faire?

Alain Robbe-Grillet : Il y a eu des auteurs dans le monde entier qui se sont avoués eux-mêmes comme ayant été influencés par le Nouveau Roman. Je pense à Peter Handke par exemple en Autriche. Je pense aussi à Julio Cortázar en Amérique du Sud et bien d'autres de par le monde. Mais créer des épigones n'est pas le but de l'artiste. Ce que je redoute le plus, ce sont les sousSimon, les sous-Robbe-Grillet, ou les sous-Duras qui affluent. Si quelqu'un doit écrire à notre suite, cela ne sera pas à notre gloire mais contre nous. Exac- 
tement comme j'estime avoir été influencé par Sartre parce que j'ai eu envie d'écrire contre lui. Et ce fait qu'on se dresse contre quelqu'un que, en quelque sorte, on admire, c'est le mouvement de la création : se dresser contre et non pas du tout aller dans la suite de...

François Jost : Je ne voudrais pas revenir sur le délire de certains théoriciens que vous avez cités tout à l'heure, mais je me suis posé différentes questions en vous entendant parler, différentes questions qui se réunissent en une seule, que je formulerai pour finir. Je me suis demandé d'abord, quand vous parliez de Ricardou, ce qu'est le rapport du théoricien à l'écrivain. Ce rapport est, au fond, assez nouveau dans la mesure où, à part les conversations de salon de Mallarmé ou de Proust, peu d'écrivains se sont trouvés sans cesse confrontés à leurs critiques. Cela me paraît une chose assez spécifique du Nouveau Roman et assez nouvelle. Quand un critique parle du Nouveau Roman, il ressent souvent une gêne dans la mesure où il a toujours un "Nouveau Romancier" en face de lui qui lui dit : «On ne peut plus dire cela parce que ce n'est plus à la mode » ou "C’est de la linguistique » etc.

Robbe-Grillet demande souvent : "Qu'est-ce qui pousse un écrivain à écrire? " Alors, je lui poserai la question : "Qu'est-ce qui pousse un critique à écrire? " C'est-à-dire, pourquoi un critique choisit-il de parler du Nouveau Roman plutôt que de Balzac ou de Flaubert? Il y a bien une raison s'il a choisi un domaine plutôt qu'un autre. On suppose souvent chez le critique une sorte d'angélisme : non seulement il devrait parler non pas de lui, mais d'un autre, mais en plus, il devrait parler de quelqu'un d'autre d'une façon qui arrange ce quelqu'un d'autre; c'est quelque chose qu'il est difficile de demander au critique. Le critique, quand il fait de la critique, cherche chez l'écrivain quelque chose qu'il ne sait pas non plus qu'il cherche. Si Ricardou trouve exactement la même chose chez Flaubert, Roussel, Mallarmé, Poe, Robbe-Grillet et Claude Simon, c'est parce qu'en fait il cherche quelque chose qu'il ne réussit pas à trouver et ce n'est pas seulement parce qu'il s'intéresse à Robbe-Grillet, à Flaubert, à Poe, à Mallarmé ou à Simon. Dans une certaine mesure, il essaie de comprendre un petit peu ce qui se passe dans sa tête.

Alors la question que peut se poser un critique, c'est de savoir - et c'est une question angoissante, qui angoisse toujours quand on écrit, il me semble - si, quand il parle d'un auteur, ce qu'il dit a quelque chance de correspondre aux textes dont il est en train de parler. C'est un problème qu'on peut se poser honnêtement parfois, et à ce moment-là on préfère sortir et faire un tour. 
Mais ce que je me demande, en ce qui concerne la critique qui a accompagné le Nouveau Roman, c'est "Est-ce qu'elle n'a pas eu, à une certaine époque, une certaine part de vérité? "Bien sûr, aujourd'hui, il est très facile de rejeter une certaine sorte de critique, que quant à moi j'ai toujours rejetée, mais est-ce que cela ne tient pas simplement au fait que le Nouveau Roman a complètement évolué. Si on a pu trouver des générateurs, ou si on a pu dire, en 1970, qu'un roman se faisait à partir, entièrement à partir, du mot " jaune » ou du mot " rouge ", est-ce que, malgré tout, cela ne correspondait pas plus ou moins à quelque chose?

Il me semble que ce qui est intéressant, précisément, dans ce mouvement du Nouveau Roman, c'est que, dans la pratique, il a réellement mis en cause ce sur quoi il s'appuyait il y a dix ans. Ce qui rend les théories d'il y a dix ans caduques, ce n'est pas que la pensée d'il y a dix ans était plus bête que celle d'aujourd'hui, c'est surtout le fait que le roman d'aujourd'hui n'est plus celui d'hier (et quand je dis roman d'aujourd'hui et celui d'hier, je me place toujours à l'intérieur du Nouveau Roman). Ce que font Nathalie Sarraute ou Claude Simon, Robert Pinget ou Alain Robbe-Grillet, c'est, bien sûr, du Sarraute, du Simon, du Pinget ou du Robbe-Grillet, mais cela n'est plus exactement du Nouveau Roman, s'il faut entendre par là une école unique et homogène. C'est la raison pour laquelle il est tout à fait stupide pour un critique de s'accrocher aux théories d'hier et d'essayer de forger une théorie unitaire comme dans les années soixante-dix. Mais, la question que je voudrais poser à tous les Nouveaux Romanciers qui sont devant nous, c'est précisément : Est-ce que vous n'avez pas l'impression que, s'il y a eu, en 1970, une critique tout à fait formaliste et un petit peu trop rigoriste, cela n'était pas aussi parce qu'elle pouvait déceler beaucoup plus facilement qu'aujourd'hui un certain nombre de choses qui appelaient une analyse structurelle?

Claude Simon : Il faudrait s'entendre sur ce " plus facilement " et sur le terme " analyse structurelle ". Je n'ai pas l'impression que mes derniers romans soient moins "structurés" que ceux d'il y a dix ou quinze ans. Mais bien sûr je peux me tromper. Seulement est-ce bien procéder à une " analyse structurelle " que de s'attacher à forger d'incessantes anagrammes avec les mots d'un texte? Et surtout à en voir là les clefs? Quelquefois cela donne des résultats piquants, quelquefois pas. Par exemple lorsque Ricardou fait de La Route des Flandres " la route des flancs ", je reste perplexe. Beaucoup plus réussie est la transformation de La Bataille de Pharsale en « La bataille de la 
phrase ". Mais où cela mène-t-il ? La bibliothèque de Genève possède des milliers d'anagrammes de Saussure. Il s'est arrêté, je crois, lorsqu'il en découvrait aussi dans des cartes postales écrites par un soldat... Mais que répondre à Ricardou lorsque celui-ci me dit : "Tu ne l'as peut-être pas voulu, mais néanmoins c'est là! » Bon, très bien. Même chose pour la couleur jaune dont il a relevé de fréquents retours dans ce même roman. C'est aussi un fait. Et puis après? Il me semble me rappeler que quelqu'un s'est escrimé au même travail sur le violet ou le mauve chez Proust. Encore une fois, tout cela me laisse assez froid et en tout cas ne me semble pas résoudre la question des structures dans un roman. À un certain moment, en mettant le poids sur la primauté du texte, Ricardou a fait œuvre de salubrité publique. Mais à trop forcer, on tombe d'un excès dans l'autre. Par contre, je vois aujourd'hui se développer ici et là, notamment avec Lucien Dällenbach, une critique qui sans cesser d'accorder au texte l'attention qu'il mérite, fait aussi appel à d'autres critères et me paraît déboucher sur des horizons beaucoup plus larges et beaucoup plus substantiels.

François Jost : J'ai écrit un article dans Poétique il y a six ans à propos de Leçon de choses où j'ai dit que ce livre me paraissait très drôle et extrêmement intéressant, mais que votre première partie qui s'appelait "Générique " était une sorte de déception par rapport à la théorie qu'on pourrait connaître9. C'est-à-dire que vous partiez d'un certain nombre de choses, une description d'un plafond, des plantes, des vagues, et puis au bout de trois pages, ce développement possible à partir d'un certain nombre de mots tournait court et vous disiez qu'on pourrait continuer indéfiniment et le roman continuait sur autre chose.

Claude Simon : Je suis désolé, Jost, mais c'est tout le contraire. Peut-être me faut-il m'expliquer sur la genèse de ce roman : à la suite d'une commande de la galerie Maeght qui avait eu l'idée d'une série de "placards " composés de petits textes illustrés par des peintres, j'ai écrit une courte description d'une pièce de ma maison de campagne à la réfection de laquelle travaillaient des maçons. Ce texte se terminait par ces lignes : "La description (la composition) peut se continuer (ou être complétée) à peu près indéfiniment selon la minutie apportée à son exécution, l'entraînement des métaphores proposées, 
l'addition d'autres objets visibles dans leur entier ou fragmentés par l'usure, le temps, un choc (soit encore qu'ils n'apparaissent qu'en partie dans le cadre du tableau), sans compter les diverses hypothèses que peut susciter le spectacle. Ainsi il n'a pas été dit, etc. "

Et puis, une fois ce texte envoyé à Maeght, je me suis dit : «Mais puisque tu as écrit cela, fais-le! Prouve que c'est possible! Continue!...» Et c'est ce que j'ai fait. Si le titre de ces premières pages est "Générique ", la suite s'intitule "Expansion ", et c'est à partir des éléments de la première description que se développent trois petites histoires, l'une racontant le travail des maçons, l'autre un épisode de la guerre (des soldats se fortifiant dans une maison en ruines dans l'attente d'une attaque ennemie), la troisième enfin mettant en scène les personnages d'une reproduction d'un tableau de Renoir encore restée punaisée sur l'un des murs de la pièce, et j'ai composé le roman en entrelaçant ces trois récits un peu à la façon dont les thèmes d'une fugue se poursuivent et se chevauchent. Aussi ai-je été heureux tout à l'heure en entendant Pinget parler de musique...

Un détail qui intéressera peut-être : alors que j'avais presque fini d'écrire ce roman, je me suis rendu compte qu'en fait, je n'avais fait que développer toutes les connotations du mot chute telles que données dans le Littré : chute d'un mur, chute de cheval, chute d'un obus, chute d'une femme, chute d'une place forte, chute du jour, etc. Mais il n’y avait rien là de concerté. Voilà comment les choses se sont passées.

François Jost : C'est justement ce que j'ai essayé de montrer.

Claude Simon : Mais vous venez de dire que le roman continuait sur autre chose que le "Générique "! Je ne comprends pas...

François Jost : Il y avait une sorte de jeu avec la critique : appeler le premier chapitre de Leçon de choses "Générique ", c'était à la fois une plaisanterie et une sorte de jeu avec toute la structuration. J'avais essayé de montrer justement que ce roman était une déception pour le " nouveau lecteur ", c'est-à-dire ce lecteur qui aurait pensé avoir tout compris du fonctionnement du Nouveau Roman, une fois pour toutes. Le problème serait : quel est le rapport entre les critiques que vous côtoyez tous les jours et votre pratique? 
Claude Simon : Il est possible qu'après avoir lu Leçon de choses, un lecteur ne comprenne pas très bien comment je suis passé de là aux Géorgiques. Pourtant, quoique ces deux textes soient très différents, leurs principes ne le sont pas tellement. Peut-être même n'aurais-je pas été capable d'écrire le dernier sans avoir écrit celui qui le précède. Mais il faudrait là de longues explications, et je ne vais pas commencer ici une conférence...

Alain Robbe-Grillet : En somme, ce qui est très bien pour Ricardou, c'est qu'il t'a déricardolisé!

Robert Pinget : J'aime beaucoup l'intervention de François Jost. Je la trouve honnête. Ils sont en train de taper sur Ricardou à bras raccourcis. Je pense qu'à un moment donné, ce critique intelligent a jeté une certaine lumière sur nos travaux et que, tout d'un coup, on ne sait pourquoi, aujourd'hui, on n'en parle plus. Il faut reconnaître que ce garçon a de la valeur.

Claude Simon : Notre ami Pinget se fait chrétiennement le défenseur du pauvre et de l'orphelin. C'est touchant et très charitable. Quoique je me demande si la vraie charité ne consisterait pas plutôt à avertir quelqu'un qui glisse sur une pente savonnée. Personne n’a jamais dit ici que les écrits théoriques de Ricardou étaient dépourvus de valeur. L’ennui, puisqu'il faut que je me répète, c'est que comme toutes les thèses poussées à l'extrême, celles qu'il a développées le conduisent dans la "pratique " à une impasse, et ceux qui le suivent avec lui. À ce titre, il représente un danger. De plus, il semble atteint depuis un certain temps d'une mégalomanie pathologique, ce qui n'arrange rien. De même que je ne lui ai pas ménagé mon approbation publiquement lorsque j'ai trouvé que ce qu'il faisait était intéressant, je lui ai dit en toute franchise (et par écrit) ce que je pense aujourd'hui. C'est tout.

Alain Robbe-Grillet : Je voudrais étendre un peu ce débat au sujet plus général des rapports de l'écrivain ou du créateur avec la critique en général. Le Nouveau Roman est un bon exemple parce qu'il a beaucoup fait parler de lui. On a énormément écrit sur le Nouveau Roman, et on a écrit les choses les plus diverses. Personnellement, j'ai toujours été très intéressé par ce qu’on écrivait sur le Nouveau Roman et par ce qu'on écrivait sur moi en particulier, bien sûr. La première chose que j'ai reconnue, c'est que le critique n'était pas un scientifique. Le critique développe ses propres fantasmes et il ne peut faire 
que cela. Le critique est une sorte d'écrivain mineur dans certains cas, majeur dans d'autres cas. Je pense à Barthes par exemple. Mais il est toujours celui qui ne fait rien d'autre que développer ses propres fantasmes. C'est-à-dire que quand j'ai parlé de la critique académique qui avait si mal reçu le Nouveau Roman, cette critique était passionnante aussi. Elle était pleine d'enseignements parce que, justement, les fantasmes de l'idéologie étaient là, présents, et c'était très intéressant de les connaître. Bien sûr pas pour corriger nos écrits, mais pour situer nous-mêmes notre propre projet. Quant à la critique universitaire ou la critique de haut niveau, se sont intéressés à moi des gens comme Blanchot et Barthes par exemple. C'est immédiatement que j'ai compris qu'il ne s'agissait pas pour eux de parler de moi, que Blanchot ne pouvait jamais faire autre chose que de parler de Blanchot et que Barthes ne pouvait parler que de Roland Barthes.

Le plus drôle, c'est qu'ils ont écrit en même temps deux articles sur Le Voyeur à une époque où j'étais à peu près inconnu, et ces deux articles sont extraordinaires! On dirait qu'ils ne parlent pas du même livre. Il y en a un des deux qui s'appelle "Littérature littérale ${ }^{10}$ ": Roland Barthes parlait du Voyeur comme s'il n'y avait pas de crime sexuel dans le livre. Il n'en parle jamais. C'est-à-dire que c'est écrit au ras des choses : objectalité, objectivité bientôt, et une sorte de réalisme qui, bien entendu, n'était pas simple. Barthes était un esprit retors. Blanchot cependant ne voyait que le crime sexuel. C'est-à-dire que le texte de Blanchot commençait par cette phrase : "D'où vient cette lumière qui illumine Le Voyeur ${ }^{11}$ ? "Cette lumière, c'était le crime même. Alors comment se fait-il que cette lumière qui illumine Le Voyeur, Barthes ne l'ait pas vue du tout? Vous savez que pendant trois ans, après la publication des Gommes, je n'ai pas dit que ce texte était une démarcation de l'Edipe roi de Sophocle. Pendant trois ans, personne ne l'a dit. Aucun critique ne l'a dit, jamais, même pas Barthes qui avait écrit un texte sur Les Gommes où il n'était jamais question ni de Sophocle ni d'Edipe. Est-ce que cela veut dire que les textes de Barthes n'étaient pas intéressants? Sûrement pas! Ils étaient passionnants! Mais ce qui est passionnant surtout, c'est qu'on puisse écrire deux choses aussi différentes. Et c'était bientôt trois quand Goldmann s'y est $\mathrm{mis}^{12}$, parce que c'était encore tout à fait d'un autre point de vue. C'est-à-dire

10. R. Barthes, «Littérature littérale », Critique, no 100-101, septembre-octobre 1955, p. 820-826.

11. M. Blanchot, «Note sur un roman : Le Voyeur », La Nouvelle Revue française, $n^{\circ}$ 31, juillet 1955, p. 105-112.

12. Voir L. Goldmann, « Nouveau Roman et réalité » dans Pour une sociologie du roman [1964], Gallimard, coll. « Tel », p. 279-324. 
que cette idée très barthésienne de la polysémie du texte, elle doit justement être mise en valeur, en lumière, non pas par un critique, car probablement luimême ne pourra développer que son propre fantasme, mais par les critiques.

J'ai beaucoup d'amitié pour Ricardou, qui était un esprit tout à fait passionnant. Il a seulement eu le tort selon moi de ne pas admettre cette polysémie. C'est-à-dire que dans les colloques qui nous concernaient à Cerisy, il avait tendance à rectifier les erreurs des autres critiques et, bientôt, nos erreurs à nous-mêmes. C'est-à-dire qu'il est un esprit dogmatique, un esprit normatif, mais la théorie n'est pas forcément normative.

Quelqu'un qui travaille sur la lumière et qui s'appuiera pour ses travaux sur la théorie corpusculaire, ne dira jamais que la théorie ondulatoire est fausse. Il admettra très bien qu'un autre physicien travaille sur la théorie ondulatoire. Or vous savez que ces deux théories sont incompatibles. Elles ne peuvent pas coller ensemble. Il est impossible qu'il y ait un monde cohérent où la lumière soit à la fois des ondes et des corpuscules. C'est impossible. Néanmoins, les physiciens de la lumière ne sont pas du tout gênés par cette contradiction. Ce que je demande pour cette critique censément scientifique, c'est qu'elle soit conforme à la science moderne, à cette science qui sait qu'elle n'est justement jamais vraie, qu'elle est simplement intéressante. Et c'est, je crois, ce qui a été le cas pour Barthes, pour Blanchot, et, dans une très large mesure, pour Ricardou aussi.

Monique Wittig : Mais pour chacun qui développe ces théories ondulatoires et corpusculaires de la lumière, chacune est juste, chacune est vraie, n'est-ce pas?

Alain Robbe-Grillet : Comment font-elles pour être justes, puisqu'elles sont incompatibles et qu'elles se détruisent mutuellement? Si la lumière est formée de particules, elle ne peut être formée d'ondes.

Monique Wittig : Et pourtant, cela est dans les deux théories.

Alain Robbe-Grillet : Non! Il n'y a jamais eu aucune expérience jusqu'à présent. Mais on en cherche, où les deux théories se vérifieraient à la fois. Pour l'instant, il faut prendre l'une ou l'autre. Elles s'excluent totalement autant que Goldmann exclut Barthes. 
Tom Bishop : Et pour la critique, Monique Wittig, est-ce qu'un point de vue critique sur tel ou tel écrivain n'en exclut pas un autre?

Monique Wittig : Non, car c'est ce que j'appelle des éléments hétérogènes. On peut avoir un point de vue hétérogène où les deux points de vue ne s'annulent pas, où chacun est juste de son point de vue.

Alain Robbe-Grillet : Je crois que c'est beaucoup plus intéressant si les deux points de vue s'annulent, c'est-à-dire si les deux pôles sont irréconciliables. Vous savez que dans la traduction de la Dialectique de Hegel, on a traduit en français thèse, antithèse, synthèse, et le mot "synthèse " marquait bien que l'esprit français ne supportait pas justement cette contradiction. Mais Aufhebung ne signifie pas du tout qu'il y aura une synthèse entre les deux, mais qu'il y aura seulement un niveau de dépassement où les deux auront concouru à autre chose. Mais le fait que dans la Dialectique, la thèse et l'antithèse soient incompatibles et qu'il y ait entre les deux une lutte à mort, c'est, à mon avis, fondamental. D'autant plus qu'il s'agit de littérature car, justement, la littérature est le lieu où ces combats entre des pôles incompatibles ont lieu.

Par exemple, à propos du Nouveau Roman, on s'est beaucoup posé la question de savoir s'il est objectif ou s'il est subjectif. Justement, ce qui est très intéressant, c'est qu'il ne peut être les deux, et que, néanmoins, les deux sont là. On peut en faire une lecture objective, comme Barthes, ou une lecture subjective, comme Blanchot. Les deux sont là à l'œuvre dans le texte. Et le texte, justement, est le lieu de cette contradiction entre des choses irréconciliables, non pas vraies toutes les deux car vraies toutes les deux, elles se toléreraient mutuellement, mais au contraire ne se tolérant pas, l'une excluant totalement l'autre. Dans cette description faite par Hegel, l'un ou l'autre doit mourir, il n'est pas question même qu'il reste en vie...

Je voudrais ajouter aussi un petit point qui concerne le problème de la conscience, car les problèmes de la conscience - la conscience du héros, la conscience des autres personnages, la conscience du narrateur, la conscience de l'écrivain et du lecteur - ce sont des problèmes très importants, parce qu'ils ont donné lieu justement à beaucoup de malentendus. Le malentendu en question, très souvent, c'est que le public s'imagine que l'écrivain lui cache quelque chose, c'est-à-dire qu'il sait ce qu'il y a dans la conscience de son personnage, mais il ne le dit pas. Ceci a été vrai dans une certaine mesure pour 
L'Étranger de Camus puisque "l'étranger " n'apparaît comme une conscience vide que dans la première partie du livre et, au contraire, comme l'a fait remarquer Nathalie Sarraute à plusieurs reprises dans ses essais théoriques et ici, la conscience de "l'étranger ", de Meursault, déborde en réalité à la fin du livre. Il déborde d'un trop-plein tandis qu'au contraire, une des choses qui a vraiment caractérisé le Nouveau Roman et qui a beaucoup choqué les lecteurs, c'est ce que ceux-ci appelaient la déshumanisation des personnages. C'est-à-dire qu'on avait affaire à des héros qui n'étaient plus des êtres humains parce qu'ils n'avaient plus de conscience. En fait, ils n'avaient plus de conscience humaniste, mais ils avaient un autre type de conscience.

Ce qui est très curieux, c'est que justement les lecteurs restent complètement conditionnés par une philosophie transcendantale qui veut que la conscience soit pleine et qu'elle diffuse du plein et du sens autour d'elle. Alors que ce que nous appelons une conscience moderne ou une conscience husserlienne, c'est une conscience vide. À l'intérieur de la conscience, il n'y a rien, dit Husserl, et la conscience, c'est seulement un mouvement de projection hors de soi, ce qu'il a appelé la phénoménologie. Ce qui est très bizarre, c'est que ce mot de phénoménologie a été employé très souvent par la critique, et constamment à tort. C'est-à-dire que pour certains, les phénomènes étaient des choses en soi, qui existaient en dehors de la conscience du sujet, et, par conséquent, on disait que l'homme était remplacé par les objets dans les livres de Robbe-Grillet. Cela a été énormément dit. Et d'autres, au contraire, supposaient que dans cette phénoménologie, la notion d'intentionnalité était malgré tout une intention qui venait d'un plein à l'intérieur de la conscience. Or qu'est-ce que c'est qu'une conscience moderne? Qu'est-ce qu'il y a dans ces personnages du Nouveau Roman qui nous réunit parfaitement, Pinget, Simon, Sarraute et tous les autres? C'est que, justement, les consciences ne sont plus des consciences pleines. Ce sont des consciences qui n'existaient que dans ce mouvement de projection hors de soi. La question qu'on peut se poser, c'est : est-ce que l'écrivain est une conscience de ce type? Aussi, je crois qu'il faut dire que la conscience du romancier est une conscience en lutte. C'est-à-dire que personne ne peut se vanter d'être vraiment une conscience husserlienne. Cela n'existe pas. Nous continuons à être des consciences transcendantes, des consciences humanistes, tandis qu'à l'intérieur de notre conscience, il y a aussi cette lutte entre la vieille conscience humaniste - Dieu, la vérité... et cette conscience purement husserlienne qui n'a jamais rien à l'intérieur de 
soi-même, mais qui est sans cesse en train de se projeter de soi-même hors de soi, de soi où il n'y a rien, vers le monde où il n'y a rien non plus.

Nathalie Sarraute : Je pense que mes amis trouvent comme moi que la grande utilité du Nouveau Roman a été une libération générale des formes et du fond du roman. Quand j'avais écrit L'Ère du soupçon en 1950, on me demandait ce que je voulais dire par «traditionnel ". C'était un mot qu'on n'employait pas. On ne savait même pas à quoi cela s'appliquait. Maintenant, on publie des romans qui ne sont pas écrits dans la forme traditionnelle, les écrivains peuvent supprimer complètement le temps chronologique, ne pas employer de noms propres, traiter le dialogue comme ils l'entendent, etc. Ce n'est pas là-dessus qu'on les juge. Cette liberté est venue, je crois, de ce mouvement du Nouveau Roman.

Les jeunes écrivains ne s’aperçoivent même pas de la liberté dont ils jouissent. Il en est ainsi d'ailleurs dans la vie privée. Les nouvelles générations ne savent pas à quelle tyrannie étaient soumises les générations précédentes. Je crois que c'est un des avantages, enfin une des réussites, du Nouveau Roman, que les formes du roman soient devenues libres. Toutes les libérations sont lentes, et on ne peut juger de leurs effets sur quelques années.

Robert Pinget : Je voudrais dire que je ne suis pas d'accord avec Alain Robbe-Grillet en ce qui concerne Husserl et le rejet de toute signification religieuse, car j'ai toujours été croyant. Je n'en parle pas dans mes écrits, mais nier la présence divine...

Alain Robbe-Grillet : Je viens de dire le contraire.

Robert Pinget : Ce n'est pas vrai.

Alain Robbe-Grillet : Je viens de dire qu'une conscience husserlienne n'existait pas. Je viens de dire, hier, qu'être un pur révolutionnaire n'existe pas et que Dieu était toujours en nous. C'est justement tout le sujet de mon propos. C'est que nous sommes le lieu de ces contradictions et que le Nouveau Roman n'est plus le roman de la liberté conquise, car la liberté ne peut être conquise une fois pour toutes puisque c'est un mouvement, elle aussi, de conquête. Nous avons besoin de Dieu pour être athées. 
Claude Simon : Je suis complètement dépassé par toute cette discussion. Par exemple, on a parlé des " personnages du Nouveau Roman ». Qu'est-ce que cela veut dire? Quels personnages, et quel "Nouveau Roman " ? Nous nageons décidément en pleine confusion... Le statut du personnage chez Robbe-Grillet, Butor, Pinget, Nathalie Sarraute ou moi-même est aussi différent qu'une carpe d'un lapin, d'un colibri ou d'un chou-fleur... Alors de quoi parle-t-on? Je voudrais le savoir, et savoir ce que je fais ici... J'ai dénoncé dans ma petite contribution à ce colloque le danger à mon avis mortel que fait périodiquement courir à l'art l'introduction d'un certain scientisme, et je viens d'écouter, à propos du roman, une véhémente discussion sur des théories de physique! Personnellement, je n'en ai que faire. J'ai essayé de dire hier que mon travail est absolument artisanal, et que le mot qui me semble convenir le mieux pour le qualifier est celui de bricolage. Toute la question pour moi consiste à commencer une phrase, la poursuivre et la terminer. À ce niveau, déjà, se posent de très complexes problèmes de structure bien difficiles à résoudre, ce à quoi je ne parviens qu'à force de ratures et de tâtonnements. Même chose pour les paragraphes, même chose pour les chapitres, et même chose pour l'ensemble d'un roman. Cela suffit amplement à ma peine, on peut m'en croire. Alors quand j'entends parler haute philosophie, lois de physique, "conscience husserlienne " ou autres sujets, je demande que l'on m'excuse, mais je dois dire humblement que c'est tout à fait en dehors de ma compétence... et de mes préoccupations.

Nathalie SARraute : Quant à moi, jamais, quand j'écris, je ne pense à la conscience, ni husserlienne, ni pleine, ni portée hors de soi, ni restée en soi. Cela m'empêcherait complètement d'écrire. 